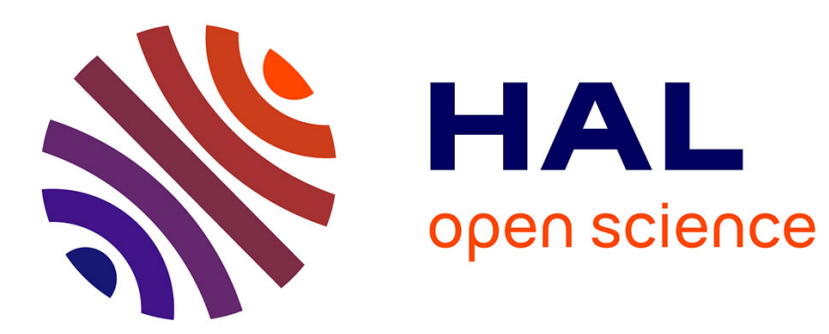

\title{
Impact of Hand Used on One-Handed Back-of-Device Performance
}

\author{
Zhuzhi Fan, Céline Coutrix
}

\section{To cite this version:}

Zhuzhi Fan, Céline Coutrix. Impact of Hand Used on One-Handed Back-of-Device Performance. Proceedings of the ACM on Human-Computer Interaction , 2020, 4, pp.1-19. 10.1145/3427316 . hal-02995376

\section{HAL Id: hal-02995376 https://hal.science/hal-02995376}

Submitted on 19 Nov 2020

HAL is a multi-disciplinary open access archive for the deposit and dissemination of scientific research documents, whether they are published or not. The documents may come from teaching and research institutions in France or abroad, or from public or private research centers.
L'archive ouverte pluridisciplinaire HAL, est destinée au dépôt et à la diffusion de documents scientifiques de niveau recherche, publiés ou non, émanant des établissements d'enseignement et de recherche français ou étrangers, des laboratoires publics ou privés. 


\title{
Impact of Hand Used on One-Handed Back-of-Device Performance
}

\author{
ZHUZHI FAN and CÉLINE COUTRIX, CNRS, Université Grenoble-Alpes, LIG, France
}

One-handed Back-of-Device (BoD) interaction proved to be desired and sometimes unavoidable with a mobile touchscreen device, for both preferred and non-preferred hands. Although users' two hands are asymmetric, the impact of this asymmetry on the performance of mobile interaction has been little studied so far. Research on one-handed BoD interaction mostly focused on the preferred hand, even though users cannot avoid in real life to handle their phone with their non-preferred hand. To better design one-handed BoD interaction tailored for each hand, the identification and measure of the impact of their asymmetry are critical. In this paper, we study the impact on the performance of the asymmetry between the preferred and the non-preferred hands when interacting with one hand in the back of a mobile touch surface. Empirical data indicates that users' preferred hand performs better than the non-preferred hand in target acquisition tasks, for both time $(+10 \%)$ and accuracy $(+20 \%)$. In contrast, for steering tasks, we found little difference in performance between users' preferred and non-preferred hands. These results are useful for the HCI community to design mobile interaction techniques tailored for each hand only when it is necessary. We present implications for research and design directly based on the findings of the study, in particular, to reduce the impact of the asymmetry between hands and improve the performance of both hands for target acquisition.

\section{CCS Concepts: • Human-centered computing $\rightarrow$ Empirical studies in HCI.}

Additional Key Words and Phrases: Mobile surfaces, Back-of-device interaction, Handedness, One-handed interaction, Steering, Target acquisition.

\section{ACM Reference Format:}

Zhuzhi Fan and Céline Coutrix. 2020. Impact of Hand Used on One-Handed Back-of-Device Performance. Author version, Author version, Article Author version (Author version 2020), 19 pages. https://doi.org/10. $1145 / 3427316$

\section{INTRODUCTION}

Back-of-device (BoD) interaction has gained a lot of interest in the $\mathrm{HCI}$ community (e.g., [20, 24, 38, $42,43,47,48,79,81])$. Indeed, BoD interaction proved to solve two important problems of mobile touch surfaces, the fat finger problem [62] (e.g., [12]) and the occlusion problem (e.g., [71]). BoD interaction also proved to solve a problem due to the most popular grasping position. Users prefer or have to hold their device vertically with a single hand $[33,49]$, with the four fingers and the palm on its back, and interact with the thumb on the screen [39]. BoD interaction helps to solve the subsequent reach-ability problem for the thumb at the front [14] (e.g., [37, 82]).

BoD interaction is now reaching the general public and the industry. E.g., the ZTE Nubia Z20 smartphone already offers a second touchscreen on the back. Apple and Microsoft are also issuing patents for interaction techniques at the BoD or devices with BoD touchscreen (e.g., [1, 35]). It is therefore timely and important that the HCI community broadens the scope of the studies of users' performance at the $\mathrm{BoD}$, in order to ensure its success.

In particular, according to an observational study of front-of-device interaction [30], users extensively use both preferred and non-preferred hands to interact with mobile surfaces. It is

Authors' address: Zhuzhi Fan, zhuzhi.fan@univ-grenoble-alpes.fr; Céline Coutrix, celine.coutrix@univ-grenoble-alpes.fr, CNRS, Université Grenoble-Alpes, LIG, 700 Avenue Centrale, Saint-Martin-d'Hères, Isère , 38401, France. 
therefore important for designers to know the difference between the hands, and if they need to adapt $\mathrm{BoD}$ interaction techniques to accommodate the hand grasping the device. However, we do not know yet the impact on BoD performance of the asymmetry between preferred (i.e. dominant) and non-preferred (i.e. non-dominant) hands. Previous work showed that the users' preferred hand can perform better than the non-preferred hand for particular tasks. For instance, Guiard found different movements resolution between preferred and non-preferred hands: the movement of the preferred hand showed higher frequency and smaller amplitudes compared to the non-preferred hand [26]. In terms of motor abilities, users' preferred hands proved to perform better for a large range of non-HCI tasks like tapping [53], drawing a circle [64], throwing [54], Annett pegboard moving task [6], handwriting [56], Fitt's task with a stylus tapping on strips, pin-to-hole transfer tasks, and circular steering tasks with stylus drawing on paper [29]. In HCI, users' preferred hand was found to tap faster on targets at the front of the device [52]. These experiments prove that the users' preferred hand are better than the non-preferred hand for these tasks. However, we do not know yet if the asymmetry impacts the tasks performed at the back of a mobile device.

In this paper, we address the following research question: What is the impact on the performance of the asymmetry between hands when interacting at the back of the device? While previous works address the sensing of the hand grasping the device $[8,16,24,44,65,73]$, the impact of the asymmetry between hands has not been measured yet.

This paper presents the following contributions:

(1) The identification and measure of the impact of the hand used on the performance of BoD target acquisition tasks: $10 \%$ faster and $20 \%$ more accurate for the preferred hand;

(2) A measure of users' performance for BoD steering tasks;

(3) A measure of the little impact of the hand used on the performance of BoD steering tasks: 3\% (resp. 2\%) slower and 8\% (resp. 16\%) more accurate in circular (resp. linear) steering tasks for the preferred hand.

The HCI community and interaction designers can benefit from these findings when designing future BoD interaction.

\section{RELATED WORK}

We present previous work that studied (1) the performance of BoD interaction on mobile surfaces, (2) the asymmetry between hands, and (3) the impact of this asymmetry on the (BoD) interaction on mobile surfaces.

First, previous work studied the performance of $\mathrm{BoD}$ interaction on mobile surfaces. For instance, Buschek et al. studied the accuracy of BoD text entry [15]. De Luca et al. found that BoD gestural authentication is slower than at the front of the device, although it was more secure [20]. BoD tapping authentication is faster than the gestural one while being as secure [40]. Combining frontand back-of-device touch input was found slower than front-of-device touch input while allowing for accurate and safe input [43]. BoD interaction allows for efficient target acquisition for very small form factors, on the contrary to front-of-device interaction [13]. Closest to our work, Wobbrock et al. studied the impact on the performance of the orientation, complexity, and support surface (i.e. front- or back-of-device), for gestures made with the thumb and the index finger, however only with the dominant hand [76].

Second, previous work studied the asymmetry between hands. The measurement of the asymmetry of motor skills between preferred and non-preferred hand began with the Annett pegboard task in 1970 [6], the first standardized task designed to analyze the motor performance of each hand [46]. In Annett's experiments [6, 7], participants moved pegs with one hand from the top row of a pegboard to the bottom row as quickly as possible. The results indicated that participants' 
preferred hands performed faster. Following Annett's pioneering work, others directly measured lateral motor performance for tasks such as tapping [53], circle making [64], and throwing [54] In these experiments, preferred hands performed better: faster [53,64] and more accurate. The preferred hand performed faster for handwriting [56] and tapping on targets on the screen of a mobile phone [52]. While Hoffmann [29] observed that participants performed significantly faster with preferred hand in Fitts' task, pin-to-hole transfer tasks, and circular steering task, it was not the case for ballistic arm movements [29].

Guiard explored the asymmetry between hands in the resolution of the movement. Guiard requested participants to manipulate a pair of potentiometric knobs, one with each hand, to produce random motion on the screen [26]. Polygraphs, recording the motion produced at each knob, revealed that the motion of users' preferred hands showed higher frequency and smaller amplitudes. Thus Guiard labeled the motion of the preferred hand as micrometric and the motion of the non-preferred hand as macrometric. These works demonstrate the difference in terms of motor abilities between the users' hands, even though some of these skills might come with training [58]

Third, previous work studied the impact of this asymmetry on the (BoD) interaction on mobile surfaces. When using a tablet with both hands holding and manipulating, Wolf et al. observed shorter movement duration for participants' preferred hand compared to non-preferred hand when making vertical drag gestures, horizontal drag gestures, and horizontal swipe gestures [78]. Wolf et al. also observed different path lengths between preferred and non-preferred hands when making BoD gestures, e.g, vertical drag, and vertical swipe. However, this study covered BoD interaction on a tablet, with both hands involved simultaneously. The study does not compare the performance of preferred vs. non-preferred hands for one-handed BoD interaction. We addressed this problem in our study.

\section{EXPERIMENT}

The goal of our experiment is twofold. First, we want to compare the performance of both hands for tasks commonly performed at the BoD. Second, we want to understand if and how other common factors, such as the difficulty of tasks and motion orientation [66, 76, 83], interact with the asymmetry of the hands at the BoD.

\subsection{Experimental tasks}

To choose our experimental tasks, we reviewed the interaction techniques proposed in related work for BoD interaction. We found taps e.g., tapping at a back keyboard [34], tapping on the phone case $[18,61,79]$, tapping on buttons [42, 47]; linear movements e.g., swiping on the phone case $[61,79]$, swiping on the rear-facing camera [81], drawing for unlocking (like Android's grid unlock on screen) [20, 41] and non-linear movements e.g., drawing a circle [80].

Based on Buxton's taxonomy of input tasks [9], taps can be considered as (1) target acquisition, and linear and non-linear movements can be considered combinations of (2) constrained linear motion and (3) constrained circular motion [3]. The parameters of these controlled tasks vary throughout the real-world task. As a consequence, to study the asymmetry between hands for BoD interaction, we decided to compare participants' two hands performance for these three controlled tasks. These controlled tasks are commonly studied in the HCI literature (e.g., [21, 76] and [2, 4]).

\subsection{Participants}

We recruited 12 participants (6 male, 6 female, $M=25.3$ y.o., $S D=2.2$ years) at the local university. Each participant received a $20 €$ voucher. All participants were right-handed. There average measured hand size lies within common measurements of human hands [36, 55]. All participants owned a multi-touch smartphone. 


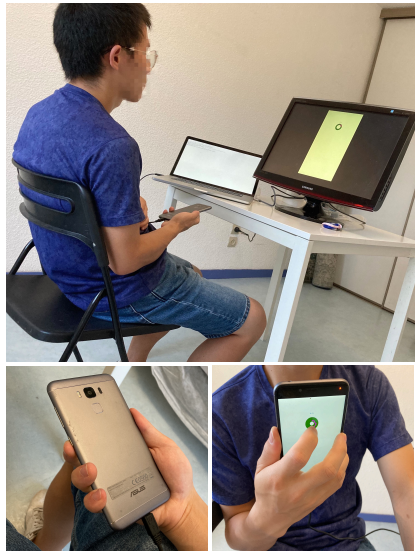

(a) Experimental setup (top), and (b) front and rear views of participants' grasp on the phone (bottom).
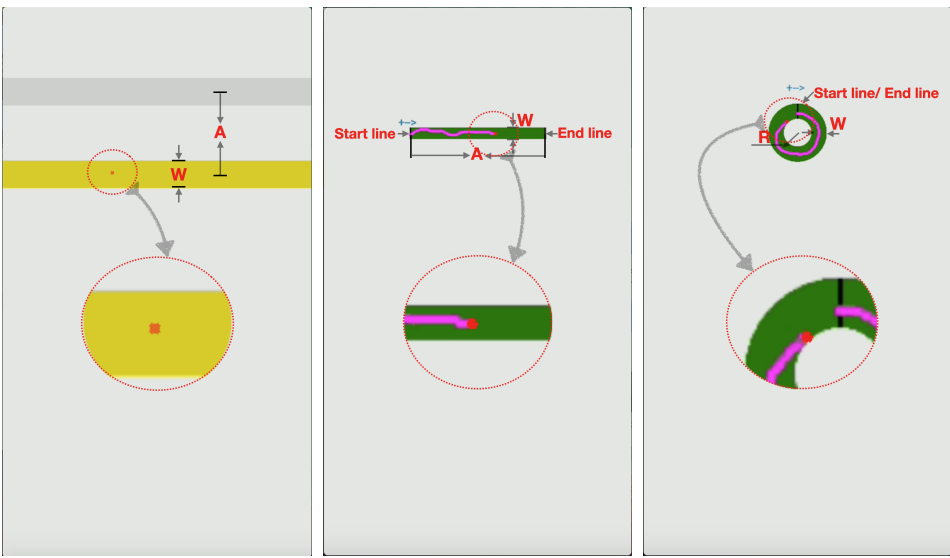

(b) Screenshot of the tar- (c) Screenshot of the con-(d) Screenshot of the conget acquisition task. "W" in- strained linear motion. "W" strained circular motion. "W" dicates the width of the indicate the tunnel width indicate the tunnel width yellow target. "A" indicates and " $A$ " its length. The blue and " $\mathrm{R}$ " its radius. The blue the movement amplitude de- arrow indicates the move- arrow indicates the movefined as the distance be- ment orientation (as in [10]). ment orientation (as in [10]) tween the centers of previ- The pink line is the finger- The pink line is the fingertip ous and next targets. Partic- tip trajectory. The black line trajectory. The black line is ipants slide their finger on are the start and end lines. the start and end line. the BoD touchscreen until the red dot enters the yellow target, and then lift their finger to validate the acquisition [76].

Fig. 1. Experimental setup and phone grasp (a) and the three tasks (b, c and d). We displayed the finger position as a red dot (b, $c$ and d). The detailed view, inside the red, dotted circle, and the indications with red labels, were not shown during the experiment.

\subsection{Apparatus}

We used an ASUS ZenFone 3 Max (5.5” screen, $1920 \times 1080$ px at $401 \mathrm{ppi}$ ) as our experimental device. To enable the BoD interaction in our experiment and keep the form factor and weight of a standard smartphone, we place the touchscreen face down (as in [41]) and separate the output display from the mobile device (as Wobbrock et al. did [76]). To display the screen of the device in front of the user, we connect the device to a laptop connected to a Samsung T240MD monitor (1920 $\times 1200$ pixels, 24" screen). The control-display gain was 2.3, as in [17]. To prevent fatigue, we first invited them to sit $60 \mathrm{~cm}$ away from the monitor, as in [22] and Figure 1(a). We then chose to set the centroid of our tasks at the height of the index's comfortable area at BoD [37] and horizontally centered (Figures 1(b-c-d)). We disabled the edge buttons of the device to avoid the accidental press.

\subsection{Procedure}

After participants filled a short demographic questionnaire, we measured the size of participants' hands, i.e. breadth, length and circumference, and the length of their index finger as in [36, 55]. In all three tasks, we presented participants' touchscreen contact location on the monitor as a red dot (Figures 1(b-c-d)). When the finger was not in contact with the touch screen, no location was shown. We asked participants to use their index finger, as it has the largest comfortable interaction 
area on the $\mathrm{BoD}$ [37]. Participants were instructed to hold the lateral sides of the device with the four other fingers, as shown in Figure 1(a). This is the most popular grasping position for BoD interaction [39].

Every participant performed all three tasks. The order of presentation of the tasks was balanced across participants according to a Latin square. Half of the participants started the experiment with their preferred hand, the other half with their non-preferred hand. After participants finished all trials for one task with one hand, they changed hand for an identical set of trials for the same task The starting hand for all three tasks was the same for a single participant.

For the target acquisition task, before the first trials of a hand, participants performed 14 practice trials, as in [76], with pseudo-randomly assigned orientation and amplitude of movement, and width of the target. The practice session was followed by the actual test. During the actual test, we pseudo-randomly ordered the different 18 conditions (2 Orientations $\times 3$ Amplitudes $\times 3$ Widths) During the actual test, for each combination of conditions, participants performed a sequence including 4 practice trials and 10 test trials. We collected only the data of the 10 test trials.

For the constrained circular (resp. linear) motion task, before the first trial of a hand, participants performed a practice session, consisting of 10 practice trials in the easiest and most difficult conditions, as in [3]. The practice session was followed by a set of pseudo-randomly ordered test trials with 24 (resp. 12) different conditions (4 (resp. 2) Orientations $\times 2$ Lengths $\times 3$ Widths), during which data was actually collected. Participants performed 5 repetitions of each condition.

Target acquisition (TA) task. We presented participants with vertical and horizontal bar targets (Figure 1(b)) common to 1-D Fitts' law experiments (e.g., [76]). Participants were instructed to slide their index finger across the touchscreen to acquire targets as quickly and accurately as possible. We used the lift-off technique to select a target, as in previous work (e.g., [69, 72, 76]). The current target was highlighted in green, while the starting position (i.e. previous target) was gray. When the dot -i.e. the touch location- was in the current target, the target turned from green to yellow (Figure 1(b)). If the finger was lifted outside the target, the current target turned from green to red and an error was recorded. Regardless of whether a given target was hit or missed, the opposite target became the active one, and the movement time of the trial was recorded. Participants were instructed to return their finger to the touch screen immediately after lifting it and to proceed to the next target (as in [76]).

Constrained linear motion (CL) task. We presented participants with vertical and horizontal tunnels to steer (Figure 1(c)). Participants were instructed to steer their index finger along the tunnel as quickly and accurately as possible, according to the orientation given by the blue arrow as shown in Figure 1(c) and done in previous work [11]. We presented in total four orientations to participants: leftward and rightward for horizontal tunnels, and upward and downward for vertical tunnels. After participants crossed the start line of the tunnel with their index finger (Figure 1(c)), the tunnel became activated, and changed color from gray to green, as a signal that the task had begun and the time was being recorded. Participants' fingertip trajectory was shown with a pink line (Figure 1(c)). When participants' finger strokes over the end line, the trial was completed, and the tunnel turned from green to yellow. As in [4], crossing the borders of the tunnel resulted in the tunnel's color turning from green to red, the cancellation of the trial, and an error was recorded. Lifting the finger after crossing the start line and before crossing the end line, but without crossing the tunnel border, resulting in an invalid trial. In this case, no error was recorded ${ }^{1}$, as in [4].

\footnotetext{
${ }^{1}$ Participants sometimes lifted their finger by mistake, but this could not be attributed to the constraints imposed on movement variability, because they did not cross the displayed tunnel border. We could not let participants continue the trial either, because we could not make sure their finger was back to the same position when lifting.
} 
Constrained circular motion (CC) task. CC (Figure 1(d)) presented the same procedure as CL, except that the tunnel to steer was circular. Participants always started at the top of the tunnel (as in $[3,4])$. They were presented with two orientations (clockwise or anticlockwise), as shown in Figure $1(\mathrm{~d})$ and done in [10].

The target acquisition task took around 20 minutes, the constrained linear motion task around 30 minutes and, the constrained circular around 30 minutes. After finishing one task with each hand, participants were asked to fill in a Raw NASA Task Load Index questionnaire [27] to measure the subjective workload imposed by completing this task. After finishing one task with both hands, participants were asked to freely comment on the task and hands used. After finishing one task, participants were allowed to take a break until they felt ready for the next task.

\subsection{Design}

For each task type, we used a fully-crossed, within-subjects factorial design with repeated measures. Independent variables were: orientation of movement, hand used, width, and amplitude (resp. length for the two steering tasks). Dependent variables were movement time (i.e. target acquisition time or steering time), error rate, and subjective workload. For target acquisition task, movement orientation, amplitude, width and hand conditions were fully-crossed. For steering tasks, movement orientation, length, width, and hand were fully crossed. The two steering task types were compared as a within-participant factor. The levels of amplitude (A) and width (W) of the target acquisition tasks were taken from a prior study [76]. For steering tasks, we found through a pilot study that the levels of tunnel length (L) and tunnel width (W) from prior work [4] lead, for one-handed BoD interaction, to error rates over $80 \%$ and extreme fatigue on fingers. To avoid this, we used the three largest tunnel widths $(2.5,3.4$, and $5.0 \mathrm{~mm})$ corresponding to their settings closest to ours, and their shortest tunnel length $(14.8 \mathrm{~mm})$. To provide with a second value for tunnel length, we double the first, shortest, tunnel length, as in [68]. Participants needed to succeed at least one time in each combination of conditions. If participants failed all trials of a given combination of conditions, these trials were rescheduled at the end of the trials, until participants succeeded once, as in $[19,70]$. Figure 2 shows the detailed experimental variables. Following previous work, we draw the following list of hypotheses:

H1: Participants' preferred hand performs faster than non-preferred hand in all conditions (task type, movement orientation, target or tunnel width, and motion amplitude or tunnel length).

H2: Participants' preferred hand performs less error than the non-preferred hand in all conditions (defined as in H1).

H3: The time difference between participants' preferred and non-preferred hand increases when the task gets more difficult (i.e. when the target gets narrower and the amplitude gets larger in target acquisition task; when the tunnel gets narrower and the tunnel gets longer is steering tasks).

H4: The difference in error rate between participants' preferred and non-preferred hand increases when the task gets more difficult (defined as in H3).

This experimental design and the planned analysis were registered ${ }^{2}$ before we collected the data.

\section{RESULTS}

To have a good estimation of the movement time, which has a skewed distribution, we used the geometric mean [59]. Shapiro-Wilk test showed that we could not assume the normality of our data $^{3}$. Therefore, we performed an aligned rank transformation [75] of the data before the repeated measures ANOVA. In the remaining of the paper, unless otherwise mentioned, all error bars and

\footnotetext{
${ }^{2}$ https://osf.io/6u3kg/?view_only=ec62d0bf6c604b95b0ddc25165249c59

${ }^{3}$ See supplementary material for analysis code and detailed results
} 


\begin{tabular}{|c|c|c|c|c|c|c|c|c|}
\hline & & \multicolumn{3}{|c|}{$\begin{array}{c}\text { Task1 } \\
\text { Target acquisition }\end{array}$} & \multicolumn{2}{|c|}{$\begin{array}{c}\text { Task2 } \\
\text { Constrained linear motion }\end{array}$} & \multicolumn{2}{|c|}{$\begin{array}{c}\text { Task3 } \\
\text { Constrained circular motion }\end{array}$} \\
\hline H & Hand & \multicolumn{7}{|c|}{ preferred hand, non-preferred hand } \\
\hline $\mathbf{0}$ & Orientation & \multicolumn{3}{|c|}{$\begin{array}{c}\text { horizontal, vertical (subsidiary } \\
\text { orientations of leftward, rightward, } \\
\text { upward, downward will also be } \\
\text { recorded) }\end{array}$} & leftwar & $\begin{array}{l}\text { d, upward, } \\
\text { ird }\end{array}$ & \multicolumn{2}{|c|}{ clockwise, anticlockwise } \\
\hline \multirow{3}{*}{ ID } & \multirow{3}{*}{$\begin{array}{l}\text { Index of } \\
\text { Difficulty }\end{array}$} & \multicolumn{3}{|c|}{$\begin{array}{c}1.32,2,2.81,3.70,4.64 \text { bits } \\
\mathrm{ID}=\log _{2}(A / W+1)[76]\end{array}$} & \multicolumn{4}{|c|}{$\begin{array}{c}0.96,4.35,5.92,8.71,11.84 \\
\mathrm{ID}=L / W[2]\end{array}$} \\
\hline & & A & Amplitude & $\begin{array}{c}144,288,576 \text { pixels } \\
(9,18,36 \mathrm{~mm})\end{array}$ & $\mathbf{L}$ & Length of th & nel & $\begin{array}{l}237,474 \text { pixels } \\
(14.8,29.6 \mathrm{~mm})\end{array}$ \\
\hline & & $\mathbf{w}$ & Width & $\begin{array}{c}24,48,96 \text { pixels } \\
(1.5,3,6 \mathrm{~mm})\end{array}$ & $\mathbf{w}$ & Width of th & nel & $\begin{array}{l}40,54,80 \text { pixels } \\
(2.5,3.4,5.0 \mathrm{~mm})\end{array}$ \\
\hline $\mathbf{T}$ & Trial & \multicolumn{3}{|c|}{$1 \ldots 10$ (as in [76]) } & \multicolumn{4}{|c|}{$1 \ldots 5$ (as in [3]) } \\
\hline
\end{tabular}

Fig. 2. Experimental variables for each task.

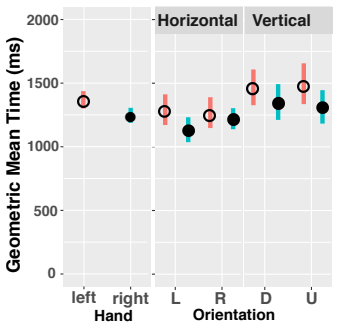

(a) Target Acquisition

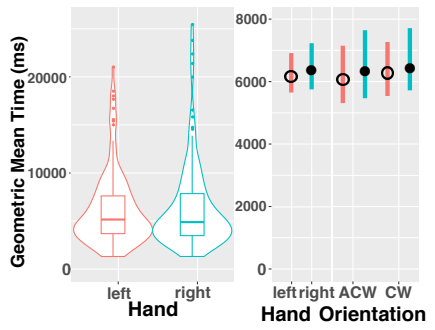

(b) Circular Steering

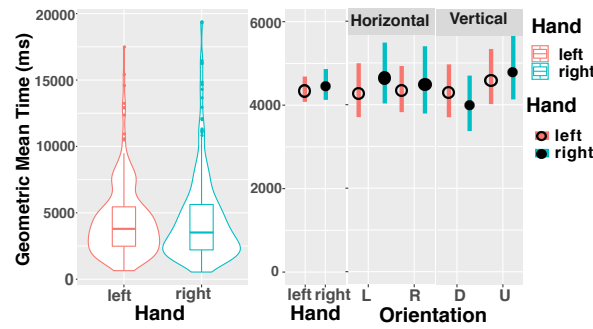

(c) Linear Steering

Fig. 3. Movement time against hand and motion orientation: (a) Target acquisition, (b) Circular Steering, and (c) Linear Steering.

CI indications are the bootstrapped $95 \%$ confidence interval, and post-hoc tests are Wilcoxon Signed-rank tests using Holm's sequential Bonferroni procedure for p-value correction.

For the target acquisition task, we calculated the standard deviation from the mean on-screen selection coordinate on the set of all 5 trials of each participant for each combination of Hand $\times A \times$ $W \times$ subsidiary orientations. As in [76], outliers were defined as trials whose selection coordinates were more than two standard deviations from the mean selection coordinate. Under this criterion, no trial was excluded.

For the two steering tasks, invalid trials caused by lifting the finger before crossing the end lines were considered outliers and excluded. We excluded 35 of such invalid trials over a total of 4326 steering trials.

For all tasks, movement time was calculated after discarding error trials, as in $[3,4,45,76]$. The data and the code used for the analysis can be found in the supplementary material, together with more detailed results.

\subsection{The preferred hand does not perform faster than non-preferred hand in all conditions ( $\mathbf{H} 1)$}

H1 is partially verified for the target acquisition task. Figure 3(a) shows that on average, participants' preferred hand $(\mathrm{M}=1240 \mathrm{~ms}, \mathrm{CI}=[1188,1306])$ performed $10 \%$ faster than the nonpreferred hand $(\mathrm{M}=1363 \mathrm{~ms}, \mathrm{CI}=[1300,1437])$. The hand has a significant impact on the movement 
time $\left(F_{(1,385)}=12.83, \mathrm{p}<0.001\right.$, non-preferred hand vs. preferred hand: $\mathrm{W}=14488, \mathrm{Z}=3.0122, \mathrm{p}<0.01$, $\mathrm{r}=0.145)$.

We also examine the movement time for different motion orientations for each hand. The interaction of hand used $\times$ motion orientation (horizontal/vertical) on movement time is not significant (Figure $3(\mathrm{a})$ ). However, the interaction of hand used $\times$ subsidiary motion orientation (leftward, rightward, downward and upward) has a significant impact on movement time $\left(F_{(3,781)}=5.03\right.$, $\mathrm{p}<0.01)$ :

With the preferred (i.e. right) hand, acquiring a target on the left was significantly faster than in any of the other three directions (leftward vs. rightward: $W=1307, Z=-5.0146, p<0.001, r=0.341$; leftward vs. downward: $\mathrm{W}=1249, \mathrm{Z}=-5.1924, \mathrm{p}<0.001, \mathrm{r}=0.353$; leftward vs. upward: $\mathrm{W}=1647, \mathrm{Z}=$ 3.9724, $\mathrm{p}<0.001, \mathrm{r}=0.270$ ). The fastest orientation with their preferred (i.e. right) hand is leftward $(M=1114 \mathrm{~ms}, \mathrm{CI}=[1037,1232])$, then rightward $(\mathrm{M}=1215 \mathrm{~ms}, \mathrm{CI}=[1138,1304])$, then upward $(M=1297 \mathrm{~ms}, \mathrm{CI}=[1186,1455]$ and slowest was downward $(\mathrm{M}=1334 \mathrm{~ms}, \mathrm{CI}=[1217,1500])$.

With the left hand, acquiring a target horizontally was significantly faster than vertically (rightward vs. downward: $\mathrm{W}=1338, \mathrm{Z}=-4.9196, \mathrm{p}<0.001, \mathrm{r}=0.335$; rightward vs. upward: $\mathrm{W}=1421, \mathrm{Z}=-4.6651$, $\mathrm{p}<0.001, \mathrm{r}=0.317$; leftward vs. downward: $\mathrm{W}=1760, \mathrm{Z}=-3.6261, \mathrm{p}<0.01, \mathrm{r}=0.247$; leftward vs. upward: $\mathrm{W}=1546, \mathrm{Z}=-4.282, \mathrm{p}<0.001, \mathrm{r}=0.291$ ). The fastest orientation with their left hand is rightward $(M=1247 \mathrm{~ms}, \mathrm{CI}=[1148,1390])$, then leftward $(\mathrm{M}=1277 \mathrm{~ms}, \mathrm{CI}=[1172,1413])$, then downward $(\mathrm{M}=1454 \mathrm{~ms}, \mathrm{CI}=[1327,1608])$, and slowest was upward $(\mathrm{M}=1475 \mathrm{~ms}, \mathrm{CI}=[1334,1655])$.

H1 is not confirmed for the steering task as a whole. The hand used had no significant impact on the movement time for overall steering tasks $\left(F_{(1,209)}=0.03, p=0.87\right)$. Even when analyzing each steering task separately, $H 1$ is not confirmed in the constrained circular motion. As shown in Figure 3(b), participants' preferred hand was on average 3\% slower $(\mathrm{M}=6407 \mathrm{~ms}, \mathrm{CI}=[5767,7240])$ than the non-preferred hand $(\mathrm{M}=6206 \mathrm{~ms}, \mathrm{CI}=[5641,6892])$. Despite this difference, the impact of Hand used on the movement time is not significant $\left(F_{(1,253)}=0.44, p=0.51\right)$. The distribution of movement time was slightly different for both hands: while the preferred hand had a lower median time (Median=4884 ms) than the non-preferred hand (Median=5155 ms), the preferred hand's movement time had more variance ( $\mathrm{SD}=4478 \mathrm{~ms}$ ) compared to the non-preferred hand $(\mathrm{SD}=3838 \mathrm{~ms})$. For constrained circular motion, no interaction of hand used $\times$ motion orientation significantly impacts the movement time. This means that the performance of each hand does not change significantly in different orientations.

H1 is not confirmed either for the constrained linear motion. Even though Figure 3(c) (left) shows that the preferred hand had a smaller median time (Median=3518 ms) than the non-preferred hand (Median=3793 ms), the preferred hand was on average $2 \%$ slower $(\mathrm{M}=4468 \mathrm{~ms}, \mathrm{CI}=[4123,4873])$ than the non-preferred hand $(\mathrm{M}=4360 \mathrm{~ms}, \mathrm{CI}=[4072,4688])$. Despite this difference, we found no significant impact of the hand used on the movement time $\left(F_{(1,517)}=2.17, p=0.14\right)$.

For constrained linear motion, the interaction between the hand and the motion orientation had a significant impact $\left(F_{(3,517)}=3.625, \mathrm{p}<0.05\right)$ : When participants interacted with their non-preferred hand, the slowest motion orientation was upward $(M=4586 \mathrm{~ms}, \mathrm{CI}=[4016,5337])$, which was significantly slower than downward $(\mathrm{M}=4271 \mathrm{~ms}, \mathrm{CI}=[3702,4967], \mathrm{W}=1878, \mathrm{Z}=3.165, \mathrm{p}<0.05, \mathrm{r}=0.37)$ and leftward ( $M=4267 \mathrm{~ms}, \mathrm{CI}=[3702,4992], \mathrm{W}=759, \mathrm{Z}=3.1145, \mathrm{p}<0.05, \mathrm{r}=0.37)$. The differences were not significant between the other orientations.

When participants interacted with their preferred hand, the fastest motion orientation was downward $(M=3974 \mathrm{~ms}, C I=[3371,4698])$, which was significantly faster than upward $(M=4784 \mathrm{~ms}$, $\mathrm{CI}=[4128,5787], \mathrm{W}=2288, \mathrm{Z}=-5.4658, \mathrm{p}<0.001, \mathrm{r}=0.46)$, and leftward $(\mathrm{M}=4641 \mathrm{~ms}, \mathrm{CI}=[4029,5487]$, $\mathrm{W}=2053, \mathrm{Z}=-4.147, \mathrm{p}<0.001, \mathrm{r}=0.35)$. The differences were not significant between other orientations. 


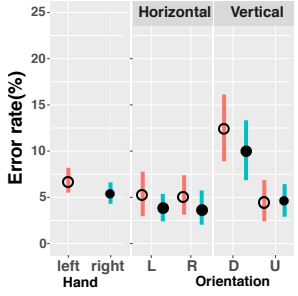

(a) Target Acquisition

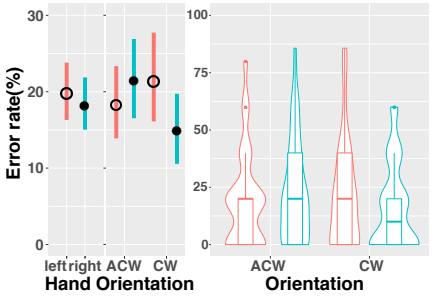

(b) Circular Steering

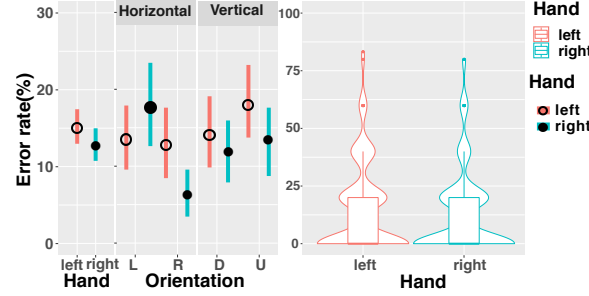

(c) Linear Steering

Fig. 4. Error rate against hand and motion orientation: (a) Target acquisition, (b) Circular Steering, and (c) Linear Steering.

\subsection{The preferred hand does not perform less error than the non-preferred hand in all conditions ( $\mathbf{H} 2)$}

H2 is partially verified for the target acquisition task. Figure 4(a) shows that the preferred hand performed at a $20 \%$ lower error rate $(M=5.42 \%, C I=[4.21,6.71])$ than the non-preferred hand $(\mathrm{M}=6.76 \%, \mathrm{CI}=[5.37,8.38])$. The impact of the hand on the error rate $\left(F_{(1,385)}=4.29, \mathrm{p}<0.05\right)$ is significant. However, the post-hoc test does not confirm the significant difference between the hands ( $W=2500.5, Z=4.7468, p=0.15, r=0.057$ ), probably as the effect size is small.

For target acquisition tasks, none of the interactions between hand used $\times$ motion orientation or hand used $\times$ subsidiary motion orientation was found significant on the error rate. This means that the accuracy of each hand does not change significantly when the motion orientation changes.

H2 is not confirmed for the steering task as a whole. The impact of the hand used on the error rate is not significant $\left(F_{(1,209)}=0.31, p=0.58\right)$. Even when analyzing each steering task separately, $\mathrm{H} 2$ is not confirmed in constrained circular motion. Figure $4(\mathrm{~b})$ shows that the preferred hand performed at an $8 \%$ lower error rate $(M=18.23 \%, C I=[15.00,21.71])$ than the non-preferred hand $(M=19.90 \%$, $\mathrm{CI}=[16.39,23.89])$. However, this is not significant $\left(F_{(1,253)}=0.4, p=0.53\right)$.

For constrained circular motion, the interaction of hand used $\times$ motion orientation do not significantly impact on the error rate. This means that the accuracy of each hand does not change significantly in different orientations.

H2 is not confirmed either in constrained linear motion. As shown in Figure 4(c), although participants' preferred hand $(\mathrm{M}=12.78 \%, \mathrm{CI}=[10.70,13.01])$ performed at a $16 \%$ lower error rate than non-preferred hand $(\mathrm{M}=15.16 \%, \mathrm{CI}=[12.98,17.48])$ the distribution of the error rate for each hand is similar, and both had a median of $0 \%$. The impact of the hand used on the error rate is not significant $\left(F_{(1,571)}=1.34, \mathrm{p}=0.25\right)$.

For constrained linear motion, the interaction between the hand used and the motion orientation had a significant impact on the error rate $\left(F_{(3,517)}=3.92, \mathrm{p}<0.01\right)$. The preferred (i.e. right) hand made the least errors rightward $(\mathrm{M}=6.94 \%, \mathrm{CI}=[4.17,10.28])$, significantly less than leftward $(\mathrm{M}=18.33 \%$, $\mathrm{CI}=[14.21,23.66] ; \mathrm{W}=494, \mathrm{Z}=4.0644, \mathrm{p}<0.001, \mathrm{r}=0.3387)$ and upward $(\mathrm{M}=13.61 \%, \mathrm{CI}=[9.72,18.33]$; $\mathrm{W}=129, \mathrm{Z}=3.072, \mathrm{p}<0.05, \mathrm{r}=0.256$ ). We found no significant differences between the other orientations for the preferred hand. When participants interacted with their non-preferred hand, we found no significant difference between any of the four orientations. 


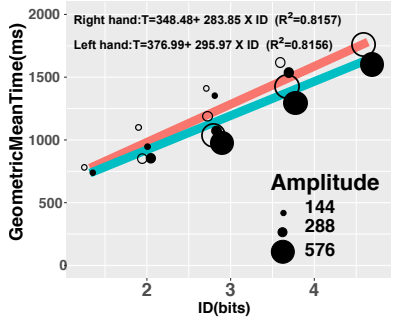

(a) Movement time against ID (Fitts' law)

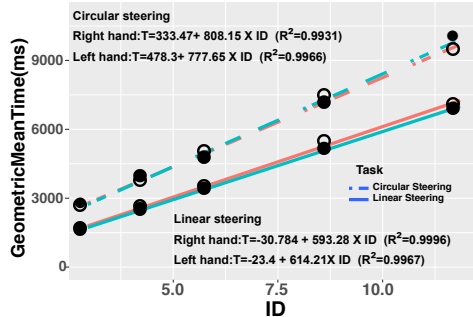

(b) Movement time against ID (Steering Law) and task type

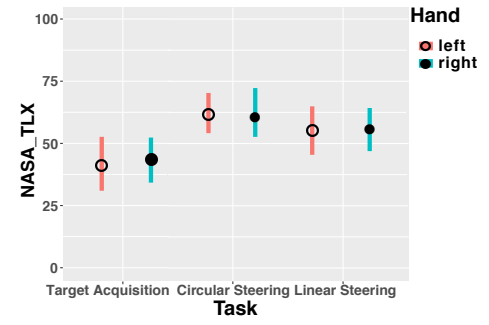

(c) NASA_TLX against task type

Fig. 5. (a) Fitts' law, (b) Steering law, and (c) Task Load Index against task types

\subsection{The time difference between the preferred and non-preferred hands does not increase when the task gets more difficult $(\mathrm{H} 3)$}

H3 is not confirmed for the target acquisition task. Figure 5(a) shows that participants performed faster with the preferred hand than non-preferred hand in all $9 \mathrm{~A} \times \mathrm{W}$ conditions, and the preferred hand is slightly faster for more difficult targets. However, the interaction between hand used $\times I D$ $\left(F_{(4,209)}=1.09, p=0.36\right)$ is not significant. The interaction of hand used $\times$ target width, and of hand used $\times$ movement amplitude were not significant either.

H3 is not confirmed for the steering task as a whole. As shown in Figure 5(b), both for circular and linear steering tasks, we found no clear difference between each hands when the ID changes. The interaction between hand used $\times I D\left(F_{(4,209)}=0.34, p=0.85\right)$ is not significant. Similarly, the interaction between hand used $\times$ tunnel length, hand used $\times$ tunnel width, hand used $\times$ tunnel width $\times$ tunnel length $\left(F_{(1,253)}=0.19, p=0.66, F_{(2,253)}=0.26, p=0.77, F_{(2,253)}=0.72, p=0.49\right.$ respectively) were not significant.

H3 is not confirmed for the constrained circular motion. None of the interaction between hand used $\times I D$, hand used $\times$ tunnel length, hand used $\times$ tunnel width, and hand used $\times$ tunnel width $\times$ tunnel length were significant $\left(F_{(4,209)}=0.68, p=0.61, F_{(1,253)}=0.38, p=0.54, F_{(2,253)}=\right.$ $0.55, p=0.58, F_{(2,253)}=2.276, p=0.10$ respectively). This shows that the hand difference does not significantly change when the task difficulty changes.

H3 is not confirmed for the constrained linear motion. The interaction of hand used $\times I D$, hand used $\times$ tunnel length, hand used $\times$ tunnel length, hand used $\times$ tunnel width, and hand used $\times$ tunnel width $\times$ tunnel length was not significant $\left(F_{(4,429)}=0.22, p=0.93, F_{(1,517)}=0.51, p=0.48\right.$, $F_{(2,517)}=0.36, p=0.70, F_{(2,517)}=0.41, p=0.67$ respectively). It means that hand difference does not significantly change with the difficulty.

\subsection{The difference in error rate between the preferred and non-preferred hand does not increase when the task gets more difficult $(\mathrm{H} 4)$}

H4 is not confirmed for the target acquisition task. We found no interaction of hand used $\times$ ID, hand used $\times$ target width, hand used $\times$ movement amplitude, hand used $\times$ movement amplitude $\times$ target width on the error rate $\left(F_{(4,209)}=2.25, p=0.06, F_{(2,385)}=0.86, p=0.42, F_{(2,385)}=2.11, p=\right.$ $0.13, F_{(4,385)}=1.81, p=0.13$ respectively).

$H 4$ is not confirmed for the steering tasks as a whole. The interaction between hand used $\times I D$ $\left(F_{(4,209)}=1.18, p=0.32\right)$ is not significant. The interaction between hand used $\times$ tunnel length, hand used $\times$ tunnel width, hand used $\times$ tunnel width $\times$ tunnel length $\left(F_{(1,253)}=2.48, p=0.12\right.$, $F_{(2,253)}=2.10, p=0.13, F_{(2,253)}=0.17, p=0.75$ respectively) were not significant either. 
H4 is not confirmed in constrained circular motion. The interaction between hand and $I D$ was significant $\left(F_{(4,209)}=2.48, p<0.05\right)$. However, the post-hoc test does not confirm the significant difference between the hands when ID value changes. None of the interaction between hand used and other factors were significant: hand used $\times$ tunnel length, hand used $\times$ tunnel width, hand used $\times$ tunnel width $\times$ tunnel length $\left(F_{(1,253)}=3.72, p=0.06, F_{(2,253)}=2.56, p=0.08\right.$, $F_{(2,253)}=1.09, p=0.34$ respectively). This shows that the hand difference does not significantly change when the difficulty changes.

H4 is not confirmed in constrained linear motion. Interactions of hand used $\times I D$, hand used $\times$ tunnel length, of hand used $\times$ tunnel width, and of hand used $\times$ tunnel width $\times$ tunnel length show no significant impact $\left(F_{(4,429)}=0.34, p=0.85, F_{(1,517)}=0.34, p=0.56, F_{(2,517)}=0.69, p=0.50\right.$, $F_{(2,517)}=0.29, p=0.75$ respectively). This means that the hand difference does not significantly change when the task difficulty changes.

\subsection{The task load is similar when interacting with the preferred vs. non-preferred hand}

Task load of target acquisition task. As shown in Figure 5(c) and by a paired t-test, the difference in task load between the preferred hand $(\mathrm{M}=43.75, \mathrm{CI}=[34.31,52.43])$ and the non-preferred hand is not significant $(\mathrm{M}=41.46, \mathrm{CI}=[31.04,52.71])$.

Task load of steering tasks. Figure 5(c) shows that participants reported a higher task load for circular $(M=61.28, C I=[55.73,67.87])$ than for linear steering $(M=55.97, C I=[49.31,62.43])$. However, this difference was not found significant. Neither the impact of hand used nor the interaction of hand used $\times$ task type show a significant impact on task load.

\subsection{Reproduction of previous results on BoD target acquisition}

In addition to answering our hypotheses, the experiment allowed us to partially reproduce previous results.

Movement time of target acquisition tasks. We first checked that the acquisition time of each hand follows Fitts' law [21]. Figure 5(a) shows the regression analysis treating all $9 \mathrm{~A} \times \mathrm{W}$ combinations separately, in order not to incorrectly increase $R^{2}$ [25]. The movement amplitude and target width significantly impact movement time $\left(F_{(2,385)}=44.89, \mathrm{p}<0.001\right.$, and $F_{(2,385)}=311.79, \mathrm{p}<0.001$ respectively). Participants were indeed faster as the target widened or as movement shortened, for both hands. We also verified that participants performed better in horizontal than in vertical orientations as in [76], and found the impact of orientation on movement time significant $\left(F_{(1,385)}=21.6\right.$, $\mathrm{p}<0.001)$. Participants performed significantly faster $(\mathrm{W}=6362, \mathrm{Z}=5.8243, \mathrm{p}<0.001, \mathrm{r}=0.280)$ horizontally $(M=1213 \mathrm{~ms}, \mathrm{CI}=[1166,1270])$ compared to vertically $(\mathrm{M}=1390 \mathrm{~ms}, \mathrm{CI}=[1322,1465])$.

Error rate of target acquisition tasks. We checked that the impact on the error rate of target width is greater than movement amplitude. Similarly to previous work [74], in our experiment, the width of target showed a significant impact $\left(F_{(2,385)}=37.13\right.$, $\left.\mathrm{p}<0.001\right)$ : participant made more errors when the target width narrowed. In contrast, the impact of the movement amplitude on the error rate is not significant $\left(F_{(2,385)}=1.19, \mathrm{p}=0.30\right)$.

We also check the significant impact of the motion orientation $\left(F_{(1,385)}=29.96, \mathrm{p}<0.001\right)$. Participants made more error vertically $(\mathrm{M}=7.82 \%, \mathrm{CI}=[6.44,9.35])$ than horizontally $(\mathrm{M}=4.35 \%, \mathrm{CI}=[3.43,5.42])$, as in [76]. The difference between these two orientations is significant $(W=3396, Z=3.7253, p<0.001$, $\mathrm{r}=0.179$ ). 


\subsection{Novel identification of the impact of subsidiary motion orientation for target acquisition tasks}

Going beyond [76], we consider subsidiary motion orientation that we introduced for target acquisition tasks. We found that participants were $3 \%$ faster $\left(F_{(3,781)}=13.23, \mathrm{p}<0.001\right)$ leftward $(\mathrm{M}=1195 \mathrm{~ms}$, $\mathrm{CI}=[1130,1281])$ than rightward $(\mathrm{M}=1231 \mathrm{~ms}, \mathrm{CI}=[1165,1314])$. We found a non-significant difference between upward $(M=1385 \mathrm{~ms}, \mathrm{CI}=[1291,1508])$ and downward $(\mathrm{M}=1394 \mathrm{~ms}, \mathrm{CI}=[1304,1496])$ movements.

We also show that the subsidiary motion orientation has a significant impact on the error rate $\left(F_{(3,781)}=21.03, \mathrm{p}<0.001\right)$. We can see in Figure 3(a) that participants made much more error downward than in the other three orientations $(M=11.11 \%$ vs. $M=4.41 \%)$. This was confirmed by post-hoc tests (downward vs. rightward: $\mathrm{W}=747.5, \mathrm{Z}=4.4644, \mathrm{p}<0.001, \mathrm{r}=0.215$; downward vs. leftward: $\mathrm{W}=567.5, \mathrm{Z}=4.7468, \mathrm{p}<0.001, \mathrm{r}=0.228$; downward vs. upward: $\mathrm{W}=553, \mathrm{Z}=5.274, \mathrm{p}<0.001, \mathrm{r}=0.254$ ). Ten (out of 12) participants told us that, when validating the target after performing a downward motion, they clearly felt their finger sliding out of the target when lifting their finger. This could explain the errors [60].

\subsection{New measure of BoD steering performance}

In addition to extending the results on target acquisition at the $\mathrm{BoD}$ [76], we also measure the movement time of steering tasks at the BoD for the first time.

Movement time of constrained motion. We first check that our data follows a Steering Law [2]. Figure 3(b) and 3(c) clearly shows that participants performed faster in linear motion ( $\mathrm{M}=4403 \mathrm{~ms}$, $\mathrm{CI}=[3939,4988])$ than in circular motion $(\mathrm{M}=6401 \mathrm{~ms}, \mathrm{CI}=[5720,7256])$ for all $\mathrm{IDs}$, as for other input devices [3, 4]. ANOVA $\left(F_{(1,209)}=212, \mathrm{p}<0.001\right)$ and a post-hoc test $(\mathrm{W}=7166, \mathrm{Z}=9.26, \mathrm{p}<0.001, \mathrm{r}=0.598)$ confirm that the task type had a significant effect on movement time.

Movement time of constrained circular motion. For constrained circular motion, we first check that the impact on movement time of tunnel length, tunnel width and interaction of tunnel length $\times$ tunnel width is significant $\left(F_{(1,253)}=641.77, \mathrm{p}<0.001, F_{(2,253)}=267.61, \mathrm{p}<0.001, F_{(2,253)}=42.72, \mathrm{p}<0.001\right.$, respectively). Participants performed faster when the tunnel is wider or shorter, as Steering Law predicts [2]. We further compared participants' performance in different motion orientation. The impact of motion orientation on movement time is not significant.

Movement time of constrained linear motion. The tunnel width and length significantly impact the movement time $\left(F_{(2,517)}=349.15, \mathrm{p}<0.001, F_{(1,517)}=1308.38, \mathrm{p}<0.001\right.$ respectively $)$. Participants were faster with a wider or shorter tunnel, as Steering Law predicts [2]. Motion orientation has a further significant impact on movement time $\left(F_{(3,517)}=6.26, \mathrm{p}<0.001\right)$. Participants were the slowest in upward steering $(M=4685 \mathrm{~ms}, \mathrm{CI}=[4225,5267])$, then leftward steering $(\mathrm{M}=4454 \mathrm{~ms}$, $\mathrm{CI}=[4017,4978])$, then rightward steering $(\mathrm{M}=4394 \mathrm{~ms}, \mathrm{CI}=[3960,4940])$, and the fastest in downward steering $(\mathrm{M}=4123 \mathrm{~ms}, \mathrm{CI}=[3714,4620])$. We found a significant difference between upward steering and rightward steering, between upward steering and downward steering, and between leftward steering and downward steering $(\mathrm{W}=3811, \mathrm{Z}=2.81, \mathrm{p}<0.05, \mathrm{r}=0.17 ; \mathrm{W}=8265, \mathrm{Z}=6.0727, \mathrm{p}<0.001$, $\mathrm{r}=0.36 ; \mathrm{W}=6884, \mathrm{Z}=3.3185, \mathrm{p}<0.0, \mathrm{r}=0.201$ respectively).

Error rate of constrained motion. Figure $4(\mathrm{~b})$ and $4(\mathrm{c})$ shows that BoD constrained circular motion causes more errors $(M=18.65 \%, C I=[15.53,22.17])$ than constrained linear motion $(M=14.05 \%$, $\mathrm{CI}=[11.80,16.67])$, as for other devices [3]. ANOVA finds a significant impact of the task on the error rate $\left(F_{(1,209)}=8.7, \mathrm{p}<0.01\right)$ and confirmed by the post-hoc test $(\mathrm{W}=3601, \mathrm{Z}=2.8058, \mathrm{r}=0.18, \mathrm{p}<0.01)$.

Error rate of constrained circular motion. We first checked the impact of tunnel width $\left(F_{(2,253)}=36.58\right.$, $\mathrm{p}<0.001)$ and tunnel length $\left(F_{(1,253)}=25.73, \mathrm{p}<0.001\right)$ on the error rate. Participants made fewer errors 
with wider and shorter tunnels, as for the stylus [4]. We further investigate the impact of motion orientation on error rate and found no significance.

Error rate of constraint linear motion. We first check the impact of the tunnel length and width on the error rate $\left(F_{(1,517)}=62.25, \mathrm{p}<0.001, F_{(2,517)}=19.52, \mathrm{p}<0.001\right.$ respectively). Participants made less errors when the tunnel was shorter and wider, as with a stylus [4]. Motion orientation also significantly impacts the error rate $\left(F_{(3,517)}=6.08, \mathrm{p}<0.001\right)$. Participants made the least errors rightward $(M=10 \%, C I=[7.36,12.92])$, significantly less than leftward $(M=16.25 \%, C I=[13.06,19.72]$, $\mathrm{W}=1509, \mathrm{p}<0.05)$ and upward $(\mathrm{M}=16.13 \%, \mathrm{CI}=[13.10,19.492], \mathrm{W}=807, \mathrm{p}<0.05)$, and slightly less than downward $(\mathrm{M}=13.50 \%, \mathrm{CI}=[10.69,16.81], \mathrm{W}=895, \mathrm{p}=0.17)$.

\section{DISCUSSION}

Partial replication and extension of previous results on BoD target acquisition. Our experiment, through its BoD target acquisition task, is a partial replication of previous work [76]. While we share most of their experimental design [76], we extend the scope of Wobbrock et al.'s experiment to non-preferred hands by exploring an additional variable (hand used). We also chose not to include other variables such as front-of-device or two-handed interactions as our primary focus was on the difference between hands, our experiment already included a lot of variables, and users preferred to use their smartphone with a single hand [33]. We also chose not to use the same, small touchpad as in [76], as nowadays users rather use a larger touchscreen like the one we use in the experiment. Other associated variables, such as the thickness of the device, were different. Our CD gain was larger (2.3 and controlled distance to screen, vs. 1.5 and non-controlled distance to screen in [76]). We tried Wobbrock et al.'s CD gain in a pilot experiment but it was too difficult.

The preferred hand of our participants was $13 \%$ faster horizontally ( $M=1156 \mathrm{~ms} v \mathrm{vs} . \mathrm{M}=1298 \mathrm{~ms}$ vertically) and performed at a $50 \%$ lower error rate horizontally ( $M=3.61 \%$ vs. $M=7.22 \%$ vertically) with the index of their preferred hand on BoD. We, therefore, found a larger difference than the BoD condition of the previous experiment [76] (resp. 10\% faster with $M=1440 \mathrm{~ms}$ vs. $M=1580 \mathrm{~ms}$ and $16 \%$ lower error rate with $M=5.65 \%$ vs. $M=6.71 \%$ ). This difference could be explained by the larger interactive area of current touch surfaces used in our experiment. We also extend Wobbrock et al.'s findings to subsidiary orientations (leftward, rightward, upward, and downward). This allows us to explain the higher error rate in vertical motion through the higher error rate in the downward motion. The error rate in the downward motion could be explained by the difficult flexion of the finger to reach bottom targets $[28,67]$.

A novel measure of steering performance at the $B o D$. We found a mean movement time of $4403 \mathrm{~ms}$ and a mean error rate of $14.5 \%$ for linear steering, and $6401 \mathrm{~ms}$ and $18.65 \%$ respectively for circular steering. Participants were faster and made fewer errors in constrained linear motion compared to constrained circular motion. Participants were faster and made fewer errors when the tunnel length shortened or when tunnel width widened. This is consistent with previous work $[3,4]$ for other devices such as the mouse, a stylus, or a touchpad. In constrained linear motion tasks, the downward motion was significantly faster than the other three motions, while the rightward motion was the least error-prone.

Impact of the hand used depends on the task. We found that the preferred hand was performing better for target acquisition tasks but in a limited way for steering tasks. To acquire a target, the preferred hand was $10 \%$ (1240 ms vs. $1363 \mathrm{~ms})$ faster and performed at a $20 \%$ (5.42\% vs. $6.76 \%$ ) lower error rate on average compared to the non-preferred hand. To steer a path, the non-preferred hand was found $2-3 \%$ faster while making $8-16 \%$ more errors. As a consequence, our hypotheses $\mathrm{H} 1$ and $\mathrm{H} 2$ are validated for target acquisition tasks but not for steering tasks. During our follow-up interview, 5/12 participants commented that they seldom steer paths. For them, no preferred hand de facto existed for steering tasks. Future work should determine if the training of each hand has 
an impact on the performance of the steering task at the BoD. Future work should also study our hypothesis that the steering task was a good candidate for a controlled task in the lab, as close as possible to trajectory-based tasks on a touchscreen in the wild.

Impact of dominance does not depend much on the difficulty. For all tasks, although the time gap between preferred and non-preferred hand widens when the difficulty increases (Figures 5(a) and 5(b)), the interaction was not significant. This applies also when separately considering the amplitude of the movement (or length of the tunnel) and the size of the target (or the tunnel). The interaction was not significant for the error rate either. Therefore our hypotheses $\mathrm{H} 3$ and $\mathrm{H} 4$ are rejected: the difference between hands does not get much larger when the task gets more difficult

Higher IDs could have revealed a higher difference between hands. In pilot experiments, we tested higher pointing and steering IDs found in previous work. However, we decided to limit the range of tested IDs for pointing and steering tasks. The first reason is that small targets (or tunnel width) lead the tasks to be very difficult. Narrowing the width of the target (or the tunnel), we found in our pilot experiment that participants can repeatedly fail all trials for a given combination The second reason is that large movement amplitude (or tunnel length) than our longest one caused extreme fatigue on participants' finger. Although our ID ranges for the two tasks are smaller than in previous work [63] [3] [4], the range of IDs we use in the paper is more realistic for BoD pointing and steering tasks.

Impact of the hand depends on the orientation. Acquiring a target opposite to the hand has more benefits for the preferred than the non-preferred hand: the difference in movement time between hands was the most significant in leftward motion. For steering tasks, the non-preferred (left) hand made significantly more error than the preferred (right) hand in the rightward direction. These imply extending the left index finger vs. flexing the right index finger.

Measure of handedness. Half of the participants performed faster and with fewer errors at the target acquisition task with their non-preferred hand. However, these participants performed $9 \%$ faster with their non-preferred $(\mathrm{M}=1176 \mathrm{~ms}, \mathrm{CI}=[1108,1265])$ compared to their preferred hand $(\mathrm{M}=1295 \mathrm{~ms}, \mathrm{CI}=[1222,1387])$. They also performed at an $11 \%$ lower error rate with their nonpreferred $(M=6.94 \%, C I=[5.09,8.89])$ compared to their preferred hand $(M=7.69 \%, C I=[5.93,9.81])$. In contrast, the other half of the participants performed $31 \%$ faster with their preferred $(M=1186 \mathrm{~ms}$, $\mathrm{CI}=[1117,1283])$ compared to their non-preferred hand $(\mathrm{M}=1550 \mathrm{~ms}, \mathrm{CI}=[1458,1676])$. They also performed at an error rate $52 \%$ lower with their preferred $(\mathrm{M}=3.15 \%, \mathrm{CI}=[2.13,4.44])$ compared to their non-preferred hand $(M=6.57 \%, C I=[4.63,8.70])$.

To further explore a possible explanation for this, we proposed an additional, optional follow-up questionnaire to participants in an attempt to more precisely define their handedness: all participants answered a 10-items Edinburgh Handedness Inventory test [50] as, e.g., in [23]. However, we found no correlation between the results of the test, and the individual target acquisition and steering performance. Another hypothesis that could be explored in future work is that participants whose hands performed more similarly are more experts at computer tasks, e.g., gamers, while participants whose non-preferred hand showed less performance are more novices.

\section{IMPLICATIONS FOR RESEARCH AND DESIGN}

Publishing "negative" results. While the impact of the hand used to interact with a mobile device had not been measured before, a lot of work addressed in the past decade the sensing of the hand grasping the device (e.g., [8, 16, 24, 44, 65, 73]). The goal of this previous research was, among others, to "optimize interfaces based on detecting handedness" [8]. Previous work sometimes questions interaction techniques designed "regardless of whether the phone is operated with the left or right hand" [44]. Previous work sometimes took for granted that "the performance of a user's dominant hand is better than that of his or her non-dominant hand" [24]. While this is true for the tasks 
presented in our related work section, many of our (pre-registered) hypotheses on the difference between the preferred and non-preferred hand for target acquisition and constraint motion at the BoD were not verified. We hope such "negative" results can help the HCI community adjust future work [31, 51].

Optimizing BoD interaction. The differences should be taken into account for the design of BoD interaction techniques. First, we found that the preferred hand was faster and performed fewer errors than the non-preferred hand for BoD target acquisition tasks. A tailored interface for the nonpreferred hand, e.g., with wider targets, can thus improve the interaction with the non-preferred hand and decrease the asymmetry of performance between the two hands.

Second, we found that, with the preferred (i.e. right) hand, acquiring a target on the opposite (i.e. left) direction was significantly faster than in any of the other three directions. We also found that the non-preferred hand was faster at acquiring targets when the movement is horizontal than vertical. These two results should be taken into account when designing for the BoD, e.g., contextual menus opening around the finger touching the screen. Such contextual UI elements should appear on the opposite side of the index finger if the preferred hand is holding and touching the back of the device, and on the same horizontal line as the index location, in the case of the non-preferred hand.

Third, we found that participants made much more error downward than in the other three orientations when acquiring targets at the $\mathrm{BoD}$. As users are less accurate when they point to the bottom of the $\mathrm{BoD}$ comfortable area of the index finger, designers should provide larger elements in this area, if they cannot be avoided. Further work should investigate the human limit of performance as a function of the location at the BoD.

Fourth, when constrained linear motion (e.g., swiping) is required at the $\mathrm{BoD}$, downward motion would be the best candidate for quick gestures, and rightward movement the best alternative for accurate gestures.

\section{CONCLUSION AND FUTURE WORK}

This paper presented a study of the impact on the performance of the hand used for one-handed interaction at the back of a mobile touch device. Empirical data indicates that users' preferred hand performs better than their non-preferred hand in target acquisition tasks, for both time $(+10 \%)$ and accuracy $(+20 \%)$. In contrast, we found little difference in the performance of steering tasks between the preferred and non-preferred hands.

Future work could investigate the impact of the validation mechanism on the difference between hands. On the one hand, as the non-preferred hand rather performs macrometric movements [26], we expect an improved validation mechanism to help the non-preferred hand. On the other hand, an improved validation mechanism could also help the preferred hand, as it did for the thumb [60]. It is however difficult to hypothesize which impact will be greater. Future work could also investigate the impact of visual attention on the difference between hands. When lacking visual attention, users leverage their proprioception. Proprioception was found to help for BoD target acquisition [77], and, for other tasks, to be impacted by handedness (e.g., [23]). In particular, the non-preferred arm can be favored [23]. Future work could also study other relevant tasks, such as pursuit (e.g., [32, 57]) or crossing tasks (e.g., [5]), and propose interaction techniques to enable easier pointing with the non-preferred hand.

\section{ACKNOWLEDGMENTS}

This work was supported by the MeMorI 80 Prime project funded by the Centre National de la Recherche Scientifique (CNRS). The authors would like to thank Katrin Wolf and François Bérard for their comments on the paper, as well as all participants. 


\section{REFERENCES}

[1] Golnaz Abdollahian and Wayne C Westerman. 2020. Device, method, and graphical user interface for force-sensitive gestures on the back of a device. US Patent 10,691,330.

[2] Johnny Accot and Shumin Zhai. 1997. Beyond Fitts' law: models for trajectory-based HCI tasks. In Proceedings of the ACM SIGCHI Conference on Human factors in computing systems. 295-302.

[3] Johnny Accot and Shumin Zhai. 1999. Performance evaluation of input devices in trajectory-based tasks: an application of the steering law. In Proceedings of the SIGCHI conference on Human Factors in Computing Systems. 466-472.

[4] Johnny Accot and Shumin Zhai. 2001. Scale effects in steering law tasks. In Proceedings of the SIGCHI conference on Human factors in computing systems. 1-8.

[5] Johnny Accot and Shumin Zhai. 2002. More than Dotting the i's - Foundations for Crossing-Based Interfaces. In Proceedings of the SIGCHI Conference on Human Factors in Computing Systems (Minneapolis, Minnesota, USA) (CHI '02). Association for Computing Machinery, New York, NY, USA, 73-80. https://doi.org/10.1145/503376.503390

[6] Marian Annett. 1970. The growth of manual preference and speed. British fournal of Psychology 61, 4 (1970), 545-558.

[7] Marian Annett. 1979. Family handedness in three generations predicted by the right shift theory. Annals of human genetics 42, 4 (1979), 479-491.

[8] Jeff Avery, Daniel Vogel, Edward Lank, Damien Masson, and Hanae Rateau. 2019. Holding patterns: detecting handedness with a moving smartphone at pickup. In Proceedings of the 31st Conference on l'Interaction Homme-Machine. $1-7$.

[9] Ronald M Baecker, Jonathan Grudin, William AS Buxton, and Saul Greenberg. 1995. Human-computer interaction: toward the year 2000. , 469-482 pages.

[10] Dong Xing Bao, Xiao Ming Li, Yi Zhong Xin, and Xiang Shi Ren. 2011. An Investigation on Tilt Angle of Touch Screen. In Information Technology for Manufacturing Systems II (Applied Mechanics and Materials, Vol. 58). Trans Tech Publications Ltd, 447-452. https://doi.org/10.4028/www.scientific.net/AMM.58-60.447

[11] Dong Xing Bao, Xiao Ming Li, Yi Zhong Xin, and Xiang Shi Ren. 2011. An Investigation on Tilt Angle of Touch Screen. In Applied Mechanics and Materials, Vol. 58. Trans Tech Publ, 447-452.

[12] Patrick Baudisch and Gerry Chu. 2009. Back-of-device interaction allows creating very small touch devices. In Proceedings of the SIGCHI Conference on Human Factors in Computing Systems. ACM, 1923-1932.

[13] Patrick Baudisch and Gerry Chu. 2009. Back-of-Device Interaction Allows Creating Very Small Touch Devices. In Proceedings of the SIGCHI Conference on Human Factors in Computing Systems (Boston, MA, USA) (CHI '09). Association for Computing Machinery, New York, NY, USA, 1923-1932. https://doi.org/10.1145/1518701.1518995

[14] Joanna Bergstrom-Lehtovirta and Antti Oulasvirta. 2014. Modeling the functional area of the thumb on mobile touchscreen surfaces. In Proceedings of the SIGCHI Conference on Human Factors in Computing Systems. ACM, 19912000.

[15] Daniel Buschek, Oliver Schoenleben, and Antti Oulasvirta. 2014. Improving Accuracy in Back-of-Device Multitouch Typing: A Clustering-Based Approach to Keyboard Updating. In Proceedings of the 19th International Conference on Intelligent User Interfaces (Haifa, Israel) (IUI '14). Association for Computing Machinery, New York, NY, USA, 57-66. https://doi.org/10.1145/2557500.2557501

[16] Lung-Pan Cheng, Hsiang-Sheng Liang, Che-Yang Wu, and Mike Y Chen. 2013. iGrasp: grasp-based adaptive keyboard for mobile devices. In Proceedings of the SIGCHI conference on human factors in computing systems. 3037-3046.

[17] Christian Corsten, Christian Cherek, Thorsten Karrer, and Jan Borchers. 2015. HaptiCase: Back-of-device tactile landmarks for eyes-free absolute indirect touch. In Proceedings of the 33rd Annual ACM Conference on Human Factors in Computing Systems. 2171-2180.

[18] Christian Corsten, Bjoern Daehlmann, Simon Voelker, and Jan Borchers. 2017. BackXPress: Using back-of-device finger pressure to augment touchscreen input on smartphones. In Proceedings of the 2017 CHI Conference on Human Factors in Computing Systems. ACM, 4654-4666.

[19] Hugo Loeches De La Fuente, Guillaume Rao, Jean-Christophe Sarrazin, Eric Berton, and Laure Fernandez. 2014. A multi-level approach to investigate the control of an input device: application to a realistic pointing task. Ergonomics 57, 9 (2014), 1380-1396.

[20] Alexander De Luca, Emanuel Von Zezschwitz, Ngo Dieu Huong Nguyen, Max-Emanuel Maurer, Elisa Rubegni, Marcello Paolo Scipioni, and Marc Langheinrich. 2013. Back-of-device authentication on smartphones. In Proceedings of the SIGCHI Conference on Human Factors in Computing Systems. ACM, 2389-2398.

[21] Paul M Fitts. 1954. The information capacity of the human motor system in controlling the amplitude of movement. Journal of experimental psychology 47, 6 (1954), 381.

[22] Jérémie Gilliot, Géry Casiez, and Nicolas Roussel. 2014. Impact of form factors and input conditions on absolute indirect-touch pointing tasks. In Proceedings of the SIGCHI Conference on Human Factors in Computing Systems. 723-732.

[23] Daniel J. Goble, Brittany C. Noble, and Susan H. Brown. 2009. Proprioceptive target matching asymmetries in lefthanded individuals. Experimental Brain Research 197 (2 July 2009), 403-408. https://doi.org/10.1007/s00221-009-1922-2 
[24] Mayank Goel, Jacob Wobbrock, and Shwetak Patel. 2012. GripSense: using built-in sensors to detect hand posture and pressure on commodity mobile phones. In Proceedings of the 25th annual ACM symposium on User interface software and technology. ACM, 545-554.

[25] Julien Gori, Olivier Rioul, Yves Guiard, and Michel Beaudouin-Lafon. 2018. The Perils of Confounding Factors: How Fitts' Law Experiments can Lead to False Conclusions. In Proceedings of the 2018 CHI Conference on Human Factors in Computing Systems. 1-10.

[26] Yves Guiard. 1987. Asymmetric division of labor in human skilled bimanual action: The kinematic chain as a model. fournal of motor behavior 19, 4 (1987), 486-517.

[27] Sandra G Hart. 2006. NASA-task load index (NASA-TLX); 20 years later. In Proceedings of the human factors and ergonomics society annual meeting, Vol. 50. Sage Publications Sage CA: Los Angeles, CA, 904-908.

[28] Khalad Hasan, Xing-Dong Yang, Hai-Ning Liang, and Pourang Irani. 2012. How to position the cursor? an exploration of absolute and relative cursor positioning for back-of-device input. In Proceedings of the 14th international conference on Human-computer interaction with mobile devices and services. 103-112.

[29] Errol R Hoffmann. 1997. Movement time of right-and left-handers using their preferred and non-preferred hands. International fournal of Industrial Ergonomics 19, 1 (1997), 49-57.

[30] Steven Hoober. 2013. How do users really hold mobile devices. (18 February 2013). https://www.uxmatters.com/mt/ archives/2013/02/how-do-users-really-hold-mobile-devices.php

[31] Kasper Hornbæk. 2015. We Must Be More Wrong in HCI Research. Interactions 22, 6 (Oct. 2015), 20-21. https: //doi.org/10.1145/2833093

[32] Yvonne Jansen, Pierre Dragicevic, and Jean-Daniel Fekete. 2012. Tangible Remote Controllers for Wall-Size Displays. In Proceedings of the SIGCHI Conference on Human Factors in Computing Systems (Austin, Texas, USA) (CHI '12) Association for Computing Machinery, New York, NY, USA, 2865-2874. https://doi.org/10.1145/2207676.2208691

[33] Amy K Karlson, Benjamin B Bederson, and Jose L Contreras-Vidal. 2006. Studies in one-handed mobile design: Habit, desire and agility. In Proc. 4th ERCIM Workshop User Interfaces All (UI4ALL). Citeseer, 1-10.

[34] Hwan Kim, Yea-kyung Row, and Geehyuk Lee. 2012. Back keyboard: a physical keyboard on backside of mobile phone using qwerty. In CHI'12 Extended Abstracts on Human Factors in Computing Systems. ACM, 1583-1588.

[35] Christian Klein, Gregg Robert Wygonik, Ricardo A Espinoza Reyes, Raymond Quan, and Sophors Khut. 2018. Gesture Language for a Device with Multiple Touch Surfaces. US Patent App. 15/257,179.

[36] Makiko Kouchi, Natsuki Miyata, and Masaaki Mochimaru. 2005. An analysis of hand measurements for obtaining representative Japanese hand models. Technical Report. SAE Technical Paper.

[37] Huy Viet Le, Sven Mayer, Patrick Bader, and Niels Henze. 2018. Fingers' Range and Comfortable Area for One-Handed Smartphone Interaction Beyond the Touchscreen. In Proceedings of the 2018 CHI Conference on Human Factors in Computing Systems. ACM, 31.

[38] Huy Viet Le, Sven Mayer, and Niels Henze. 2018. InfiniTouch: Finger-aware interaction on fully touch sensitive smartphones. In The 31st Annual ACM Symposium on User Interface Software and Technology. ACM, 779-792.

[39] Songil Lee, Gyouhyung Kyung, Jungyong Lee, Seung Ki Moon, and Kyoung Jong Park. 2016. Grasp and index finger reach zone during one-handed smartphone rear interaction: effects of task type, phone width and hand length Ergonomics 59, 11 (2016), 1462-1472.

[40] Luis A. Leiva and Alejandro Català. 2014. BoD Taps: An Improved Back-of-Device Authentication Technique on Smartphones. In Proceedings of the 16th International Conference on Human-Computer Interaction with Mobile Devices Services (Toronto, ON, Canada) (MobileHCI '14). Association for Computing Machinery, New York, NY, USA, 63-66. https://doi.org/10.1145/2628363.2628372

[41] Luis A Leiva and Alejandro Català. 2014. BoD taps: an improved back-of-device authentication technique on smartphones. In Proceedings of the 16th international conference on Human-computer interaction with mobile devices \& services. ACM, 63-66.

[42] Kevin A Li, Patrick Baudisch, and Ken Hinckley. 2008. Blindsight: eyes-free access to mobile phones. In Proceedings of the SIGCHI Conference on Human Factors in Computing Systems. ACM, 1389-1398.

[43] Markus Löchtefeld, Christoph Hirtz, and Sven Gehring. 2013. Evaluation of hybrid front-and back-of-device interaction on mobile devices. In Proceedings of the 12th International Conference on Mobile and Ubiquitous Multimedia. ACM, 17.

[44] Markus Löchtefeld, Phillip Schardt, Antonio Krüger, and Sebastian Boring. 2015. Detecting users handedness for ergonomic adaptation of mobile user interfaces. In Proceedings of the 14th International Conference on Mobile and Ubiquitous Multimedia. ACM, 245-249.

[45] I Scott MacKenzie and R William Soukoreff. 2003. Card, English, and Burr (1978) 25 years later. In CHI'03 Extended Abstracts on Human Factors in Computing Systems. 760-761.

[46] Chris McManus. 2019. Half a century of handedness research: Myths, truths; fictions, facts; backwards, but mostly forwards. Brain and Neuroscience Advances 3 (2019), 2398212818820513 
[47] Takashi Miyaki and Jun Rekimoto. 2009. GraspZoom: zooming and scrolling control model for single-handed mobile interaction. In Proceedings of the 11th International Conference on Human-Computer Interaction with Mobile Devices and Services. ACM, 11.

[48] Mohammad Faizuddin Mohd Noor, Andrew Ramsay, Stephen Hughes, Simon Rogers, John Williamson, and Roderick Murray-Smith. 2014. 28 frames later: predicting screen touches from back-of-device grip changes. In Proceedings of the SIGCHI Conference on Human Factors in Computing Systems. ACM, 2005-2008.

[49] Alexander Ng, Stephen A Brewster, and John Williamson. 2013. The impact of encumbrance on mobile interactions. In IFIP Conference on Human-Computer Interaction. Springer, 92-109.

[50] Richard C Oldfield et al. 1971. The assessment and analysis of handedness: the Edinburgh inventory. Neuropsychologia 9, 1 (1971), 97-113.

[51] Antti Oulasvirta and Kasper Hornbæk. 2016. HCI Research as Problem-Solving. In Proceedings of the 2016 CHI Conference on Human Factors in Computing Systems (San Jose, California, USA) (CHI '16). Association for Computing Machinery, New York, NY, USA, 4956-4967. https://doi.org/10.1145/2858036.2858283

[52] Keith B Perry and Juan Pablo Hourcade. 2008. Evaluating one handed thumb tapping on mobile touchscreen devices. In Proceedings of graphics interface 2008. 57-64.

[53] Michael Peters. 1980. Why the preferred hand taps more quickly than the non-preferred hand: three experiments on handedness. Canadian fournal of Psychology/Revue canadienne de psychologie 34, 1 (1980), 62.

[54] Michael Peters. 1990. Subclassification of non-pathological left-handers poses problems for theories of handedness. Neuropsychologia 28, 3 (1990), 279-289.

[55] Alan Poston. 2000. Human engineering design data digest. Washington, DC: Department of Defense Human Factors Engineering Technical Advisory Group (2000), 61-75.

[56] KA Provins and J Magliaro. 1989. Skill, strength, handedness, and fatigue. fournal of Motor Behavior 21, 2 (1989), 113-121.

[57] Simon Robinson, Céline Coutrix, Jennifer Pearson, Juan Rosso, Matheus Fernandes Torquato, Laurence Nigay, and Matt Jones. 2016. Emergeables: Deformable Displays for Continuous Eyes-Free Mobile Interaction. In Proceedings of the 2016 CHI Conference on Human Factors in Computing Systems (San Jose, California, USA) (CHI '16). Association for Computing Machinery, New York, NY, USA, 3793-3805. https://doi.org/10.1145/2858036.2858097

[58] Taebeum Ryu, Jihyoun Lim, Joobong Song, Myung Hwan Yun, and Moonsoo Shin. 2013. Performance comparison between the preferred right and preferred left hands in text entry using Qwerty touch keyboard smartphones. International Journal of Industrial Ergonomics 43, 5 (2013), 400-405.

[59] Jeff Sauro and James R Lewis. 2010. Average task times in usability tests: what to report?. In Proceedings of the SIGCHI Conference on Human Factors in Computing Systems. 2347-2350.

[60] Adriano Scoditti, Thomas Vincent, Joëlle Coutaz, Renaud Blanch, and Nadine Mandran. 2011. TouchOver: Decoupling Positioning from Selection on Touch-based Handheld Devices. In IHM 2011 - Conférence Francophone sur l'Interaction Homme-Machine. ACM, Nice - Sophia Antipolis, France, 37-40. https://doi.org/10.1145/2044354.2044362 Session: Interagir sur supports mobiles (article No 6).

[61] Shaikh Shawon Arefin Shimon, Sarah Morrison-Smith, Noah John, Ghazal Fahimi, and Jaime Ruiz. 2015. Exploring user-defined back-of-device gestures for mobile devices. In Proceedings of the 17th International Conference on HumanComputer Interaction with Mobile Devices and Services. 227-232.

[62] Katie A Siek, Yvonne Rogers, and Kay H Connelly. 2005. Fat finger worries: how older and younger users physically interact with PDAs. In IFIP Conference on Human-Computer Interaction. Springer, 267-280.

[63] R William Soukoreff and I Scott MacKenzie. 2004. Towards a standard for pointing device evaluation, perspectives on 27 years of Fitts' law research in HCI. International journal of human-computer studies 61, 6 (2004), 751-789.

[64] SM Tapley and MP Bryden. 1985. A group test for the assessment of performance between the hands. Neuropsychologia 23, 2 (1985), 215-221.

[65] Brandon T Taylor and V Michael Bove Jr. 2009. Graspables: grasp-recognition as a user interface. In Proceedings of the SIGCHI Conference on Human Factors in Computing Systems. 917-926.

[66] Namal Thibbotuwawa, Ravindra S Goonetilleke, and Errol R Hoffmann. 2012. Constrained path tracking at varying angles in a mouse tracking task. Human Factors 54, 1 (2012), 138-150.

[67] Matthieu B Trudeau, Tawan Udtamadilok, Amy K Karlson, and Jack T Dennerlein. 2012. Thumb motor performance varies by movement orientation, direction, and device size during single-handed mobile phone use. Human factors 54 , 1 (2012), 52-59.

[68] M Eduard Tudoreanu and Eileen Kraemer. 2008. A study of the performance of steering tasks under spatial transformation of input. In Proceedings of the 46th Annual Southeast Regional Conference on XX. 340-345.

[69] Daniel Vogel and Patrick Baudisch. 2007. Shift: a technique for operating pen-based interfaces using touch. In Proceedings of the SIGCHI conference on Human factors in computing systems. 657-666. 
[70] David Way and Joseph Paradiso. 2014. A usability user study concerning free-hand microgesture and wrist-worn sensors. In 2014 11th International Conference on Wearable and Implantable Body Sensor Networks. IEEE, 138-142.

[71] Daniel Wigdor, Clifton Forlines, Patrick Baudisch, John Barnwell, and Chia Shen. 2007. Lucid touch: a see-through mobile device. In Proceedings of the 20th annual ACM symposium on User interface software and technology. ACM, 269-278.

[72] Daniel Wigdor, Darren Leigh, Clifton Forlines, Samuel Shipman, John Barnwell, Ravin Balakrishnan, and Chia Shen. 2006. Under the table interaction. In Proceedings of the 19th annual ACM symposium on User interface software and technology. 259-268.

[73] Raphael Wimmer and Sebastian Boring. 2009. HandSense: discriminating different ways of grasping and holding a tangible user interface. In Proceedings of the 3rd International Conference on Tangible and Embedded Interaction. 359-362.

[74] Jacob O Wobbrock, Edward Cutrell, Susumu Harada, and I Scott MacKenzie. 2008. An error model for pointing based on Fitts' law. In Proceedings of the SIGCHI conference on human factors in computing systems. 1613-1622.

[75] Jacob O Wobbrock, Leah Findlater, Darren Gergle, and James J Higgins. 2011. The aligned rank transform for nonparametric factorial analyses using only anova procedures. In Proceedings of the SIGCHI conference on human factors in computing systems. 143-146.

[76] Jacob O Wobbrock, Brad A Myers, and Htet Htet Aung. 2008. The performance of hand postures in front-and back-of-device interaction for mobile computing. International Journal of Human-Computer Studies 66, 12 (2008), 857-875

[77] Katrin Wolf, Christian Müller-Tomfelde, Kelvin Cheng, and Ina Wechsung. 2012. Does Proprioception Guide Back-ofDevice Pointing as Well as Vision?. In CHI '12 Extended Abstracts on Human Factors in Computing Systems (Austin, Texas, USA) (CHI EA '12). Association for Computing Machinery, New York, NY, USA, 1739-1744. https://doi.org/10. 1145/2212776.2223702

[78] Katrin Wolf, Robert Schleicher, and Michael Rohs. 2014. Ergonomic characteristics of gestures for front-and backof-tablets interaction with grasping hands. In Proceedings of the 16th international conference on Human-computer interaction with mobile devices \& services. 453-458.

[79] Pui Chung Wong, Hongbo Fu, and Kening Zhu. 2016. Back-Mirror: back-of-device one-handed interaction on smartphones. In SIGGRAPH ASIA 2016 Mobile Graphics and Interactive Applications. ACM, 10.

[80] Huiyue Wu and Liuqingqing Yang. 2019. User-Defined Gestures for Dual-Screen Mobile Interaction. International Journal of Human-Computer Interaction (2019), 1-15.

[81] Xiang Xiao, Teng Han, and Jingtao Wang. 2013. LensGesture: augmenting mobile interactions with back-of-device finger gestures. In Proceedings of the 15th ACM on International conference on multimodal interaction. ACM, 287-294.

[82] Hyunjin Yoo, Jungwon Yoon, and Hyunsoo Ji. 2015. Index finger zone: Study on touchable area expandability using thumb and index finger. In Proceedings of the 17th International Conference on Human-Computer Interaction with Mobile Devices and Services Adjunct. ACM, 803-810.

[83] Xiaolei Zhou, Xiangshi Ren, and Yue Hui. 2008. Effect of start position on human performance in steering tasks. In 2008 International Conference on Computer Science and Software Engineering, Vol. 2. IEEE, 1098-1101.

Received July 2020; revised August 2020; accepted September 2020 